\title{
BLOQUEO AURICULOVENTRICULAR FETAL COMPLETO: ENFOQUE DIAGNÓSTICO Y TERAPÉUTICO. REPORTE DE CASO EN BOGOTÁ, COLOMBIA, Y REVISIÓN DE LA LITERATURA
}

\section{Complete foetal atrioventricular block: diagnostic and therapeutic approach. Case report in Bogotá, Colombia, and review of the literature}

\author{
Diana Cecilia Poveda-Rojas, $M D^{1}$; Natalia Vélez-Tirado, $M D^{2}$; \\ Leonardo Bonilla-Cortés, $\mathrm{MD}^{3}$; Juan Pablo Rozo-Galindo, $\mathrm{MD}^{4}$
}

Recibido: junio 13/16 - Aceptado: noviembre 28/17

\section{RESUMEN}

Objetivo: reportar un caso de bloqueo auriculoventricular completo congénito y realizar una revisión de la literatura del diagnóstico y tratamiento.

Materiales y métodos: se reporta el caso de una gestante de 27 años quien consulta a un hospital general de alto nivel de complejidad, con un embarazo de 33 semanas, con feto único, con diagnóstico de bloqueo auriculoventricular completo y cardiomiopatía dilatada secundaria. Se inició manejo prenatal con betamimético con pobre respuesta, por lo cual fue necesario finalizar el embarazo. El recién nacido requiere implantación de marcapasos ventricular en el primer día de vida con excelentes resultados en el seguimiento a un año. Se realiza revisión de la literatura publicada en las bases de datos: Medline vía PubMed, Lilacs y SciELO mediante los términos: "fetal complete atrioventricular block", "congenital

1 Ginecoobstetra, Universidad Militar Nueva Granada, Bogotá (Colombia). dianeporo718@hotmail.com

2 Pediatra, Universidad Militar Nueva Granada, Bogotá (Colombia).

3 Especialista en Medicina Materno-Fetal, Unidad de Ginecología y Obstetricia. Hospital Universitario Clínica San Rafael, Bogotá (Colombia).

4 Especialista en Cardiología Pediátrica-Hemodinamista, Unidad de Pediatría, Hospital Universitario Clínica San Rafael, Bogotá (Colombia). complete heart block", con límites de año de 2000 a 2016, en español e inglés.

Resultados: se obtuvieron 21 publicaciones; siete reportes de caso, diez revisiones de literatura, cuatro estudios de cohorte. El diagnóstico se basa en la ecocardiografía fetal para determinar el intervalo PR, la relación auriculoventricular y la detección de anomalías intracardiacas, entre las que se encuentran: regurgitación valvular, hiperecogenicidad miocárdica/valvular-fibroelastosis endocárdica, contracciones atriales prematuras y efusión pericárdica. Respecto al tratamiento prenatal, los medicamentos más utilizados son los corticoesteroides y los betamiméticos. El tratamiento de la bradiarritimia severa refractaria neonatal puede requerir la implantación de un marcapaso como manejo definitivo.

Conclusión: el bloqueo AV congénito de tercer grado requiere diagnóstico temprano y tratamiento oportuno, ya que se acompaña de alta morbimortalidad perinatal. Se requieren estudios con mayor calidad metodológica que permitan avalar otras opciones y esquemas terapéuticos prometedores.

Palabras clave: bradiarritmia, bloqueo atrioventricular, lupus eritematoso sistémico. 


\section{ABSTRACT}

Objective: To report a case of complete congenital atrioventricular block and to review the literature on diagnosis and treatment.

Materials and methods: Case report of a 27-yearold pregnant woman who came to a high complexity general hospital with a 33-week singleton gestation and a diagnosis of complete atrioventricular block and secondary dilated cardiomyopathy. Response to initial prenatal management with beta-mimetic therapy was poor, and the woman had to be taken to Cesarean section. The newborn required implantation of a ventricular pacemaker on the first day of life, with excellent results at 1-year follow-up. A review of the literature published in Medline, Lilacs and SciELO databases was conducted using the terms "foetal complete atrioventricular block", "congenital complete heart block," limited to articles published between 2000 and 2016 in Spanish and English.

Results: Overall, 21 publications were retrieved: seven case reports, ten reviews of the literature and four cohort studies. Diagnosis is based on the foetal echocardiographic scan to determine the PR interval and the atrioventricular ratio, and to detect intracardiac abnormalities, including valvular regurgitation, myocardial/valvular hyperechogenicity, endocardial fibroelastosis, premature atrial contractions, and pericardial effusion. In terms of prenatal treatment, corticosteroids and beta-mimetics are the most widely used medications. Treatment of severe neonatal refractory bradyarrhythmia may require pacemaker implantation as definitive management.

Conclusion: Congenital third-degree AV bock requires early diagnosis and timely treatment, because associated perinatal morbidity and mortality are high. Studies with better methodological quality are needed in order to endorse other promising therapeutic options and approaches.

Key words: Bradyarrhythmia, atrioventricular block, systemic lupus erythematosus.

\section{INTRODUCCIÓN}

La bradiarritmia fetal se define como la presencia de frecuencia cardiaca menor a 110 latidos por minuto (lpm) durante 10 minutos en ausencia de pérdida de bienestar fetal (1). Las bradiarritmias fetales incluyen: la bradicardia sinusal, las extrasístoles auriculares no conducidas asociadas a bigeminismos, y los bloqueos auriculoventriculares de segundo y tercer grado; no se incluye en esta clasificación el bloqueo auriculoventricular (AV) de primer grado por no producir bradicardia. La diferencia entre cada uno de los tipos de bloqueos AV radica en la relación entre la aurícula y el ventrículo; en el bloqueo de primer grado el segmento PR se encuentra prolongado en la totalidad de los impulsos auriculares conducidos de forma exitosa al ventrículo; en el bloqueo AV de segundo grado, el segmento PR se prolonga con cada latido hasta que hay un impulso auricular que no logra ser transmitido al ventrículo y, por último, en el bloqueo AV grado III hay evidencia de disociación auriculoventricular (2). La frecuencia cardiaca puede ayudar a determinar la etiología del bloqueo; se ha visto que la frecuencia entre 80-100 lpm es común en la bradicardia sinusal; la frecuencia entre 60-80 lpm es más frecuente en el bloqueo AV grados II y III, al igual que ante la presencia de extrasístoles auriculares no conducidas asociadas a bigeminismos; finalmente, la frecuencia cardiaca menor a $60 \mathrm{lpm}$ casi siempre se asocia a bloqueo AV grado III (3).

Se ha reportado que los bloqueos $\mathrm{AV}$ constituyen el $2 \%$ de todas las arritmias fetales, siendo el $50 \%$ causadas por cardiopatías congénitas (isomerismo atrial izquierdo, transposición de grandes arterias y canal auriculoventricular), y el otro $50 \%$ de origen inmunológico asociado a enfermedades de tejido conectivo materno $(1,4,5)$. Respecto al origen inmunológico, este es causado por anticuerpos anti-Ro y anti-LA maternos en un 85-90\%, siendo estos anticuerpos expresados en enfermedades del colágeno como lupus eritematoso sistémico y síndrome de Sjögren (6). Un porcentaje de las 
maternas portadoras de anticuerpos anti-Ro y anti-La no presentan síntomas de la enfermedad, por lo tanto hay un grupo de mujeres que quedará en embarazo sin saber el riesgo que corre el feto (7); afortunadamente, solo en el $2 \%$ de las madres seropositivas los fetos presentarán afección cardiaca, particularmente del sistema de conducción (5, 7). Para comprender la fisiopatología del bloqueo AV congénito es necesario entender el papel fundamental de los anticuerpos anti-Ro y anti-La, los cuales se dirigen contra antígenos del complejo de ribonucleoproteína presentes en las células de conducción cardiaca y miocitos cardiacos ubicados en el citoplasma (8). La unión de inmunoglobulina tipo IgG a estos antígenos desencadena tres mecanismos patológicos: 1) mimetismo molecular (regulación a la baja de los canales tipo L y T, alteración de homeostasis de calcio intracelular); 2) inducción de apoptosis en miocitos y células de conducción cardiaca; 3) inducción de fibrosis (opsonización de cardiomiocitos y sistema de conducción) que genera eliminación de las células de conducción cardiaca, y produce inflamación y fibrosis, en especial del nodo sinoauricular (9). Los anticuerpos pueden ser transferidos en cualquier momento de la gestación (10), iniciando su paso placentario a las 12 semanas; el periodo de mayor vulnerabilidad oscila entre las 16 y 24 semanas, cuando suele haber un pico en la concentración de anticuerpos, lo que explica el hecho de que más del $80 \%$ de los casos de bloqueo AV congénito sean diagnosticados antes de la semana $30(4,10)$.

Cuando el bloqueo AV se asocia a cardiopatía congénita el pronóstico es desfavorable, con una mortalidad mayor del 50-80\% en el primer año de vida; el isomerismo atrial izquierdo es la malformación que mayor mortalidad produce (11). Lo anterior contrasta con el bloqueo de origen inmunológico cuya mortalidad intrauterina es de 6\%, mortalidad global del 16-19\%, y cuando se asocia a fibroelastosis endocárdica es del 69\%, lo cual requiere el uso de marcapasos hasta en un $70 \%$ de los casos durante los 10 primeros años de vida (11,
12). El riesgo de recurrencia en gestaciones futuras es del 10-17,4\% (13).

Por ser una entidad poco frecuente es importante el reconocimiento temprano y el manejo oportuno por parte del obstetra buscando mejorar el desenlace del recién nacido. Por esta razón se presenta el caso de una gestante que cursa con un embarazo en el cual el feto es portador de bloqueo AV grado III, que requiere la implantación temprana de marcapasos en el recién nacido; el objetivo fue realizar una revisión de la literatura en donde se busca resaltar los aspectos más importantes del diagnóstico y tratamiento.

\section{REPORTE DE CASO}

Paciente de 27 años, gesta 2, abortos 1, vivos 0, que ingresa con edad gestacional de 33 semanas a la unidad de obstetricia del Hospital Universitario Clínica San Rafael (HUCSR), institución de cuarto nivel de complejidad ubicada en Bogotá, que atiende pacientes de alto riesgo materno-fetal pertenecientes al régimen contributivo en el sistema de seguridad social en Colombia. La paciente fue remitida de consulta externa por bradicardia fetal y sospecha diagnóstica de bloqueo AV fetal grado II desde la semana 25 de gestación, para valoración y manejo por medicina materno-fetal y cardiología pediátrica.

Al ingreso al HUCSR se realizan pruebas de bienestar fetal las cuales son normales; posteriormente se realiza ecografía de detalle en la cual no se observan signos de hidrops fetal ni otras alteraciones anatómicas. Finalmente, se realiza ecocardiograma fetal donde se encuentra situs solitus, situs vascular normal, eje cardiaco de $45^{\circ}$, relación cardiotorácica de 0,55, corte de cuatro cámaras normal, concordancia auriculoventricular y ventriculoarterial, inserción valvular normal, foramen oval permeable, flujo hacia aurícula izquierda, movimientos de paredes libres de ventrículos de amplitud normal; se observa imagen hiperecogénica a nivel del nodo sinoauricular sugestiva de fibroelastosis endocárdica (figura 1). No se evidencia ritmo sinusal, se documenta disociación 


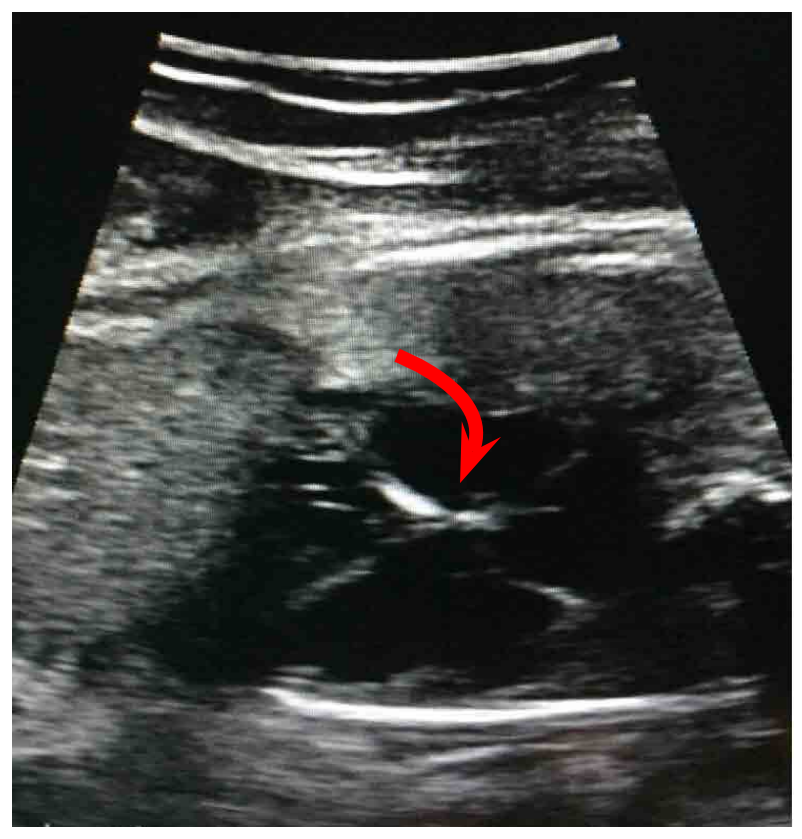

Figura 1. Fotografía del corte de cuatro cámaras donde se evidencia hiperecogenicidad a nivel del nodo sinoauricular y de la válvula tricúspidea, secundaria a la calcificación por el proceso de cicatrización, indicativa de fibroelastosis endocárdica

auriculoventricular en Modo M (figura 2), con frecuencia cardiaca ventricular de $50 \mathrm{lpm}$ y frecuencia auricular de $135 \mathrm{lpm}$, segmentos PR variables, e istmo aórtico con flujo anterógrado. No hay evidencia de disfunción diastólica dada por ductus venoso normal.

Se solicita perfil inmunológico a la madre y se inicia manejo con dexametasona $4 \mathrm{mg}$ vía oral día. Se recibe reporte de anticuerpos antinucleares (ANAS) positivos 1/80 con patrón moteado, con lo cual se hace diagnóstico de síndrome similar al lupus. Estudios de extensión reportan anticoagulante lúpico negativo, anti-Smith (anti-SM) y antirribonucleoproteína (anti-RNP) negativos, anti-Ro positivo y anti-La negativo. Dada la bradicardia fetal marcada (menor a 60 lpm) y el hallazgo de disociación auriculoventricular, se hace el diagnóstico de bloqueo auriculoventricular grado III. Se inicia terbutalina vía oral $5 \mathrm{mg}$ cada 4 horas, la madre se hospitaliza para maduración pulmonar y seguimiento con perfil biofísico cada 48 horas buscando alcanzar los 2500 mg en el feto. Posterior al inicio de la terbutalina se

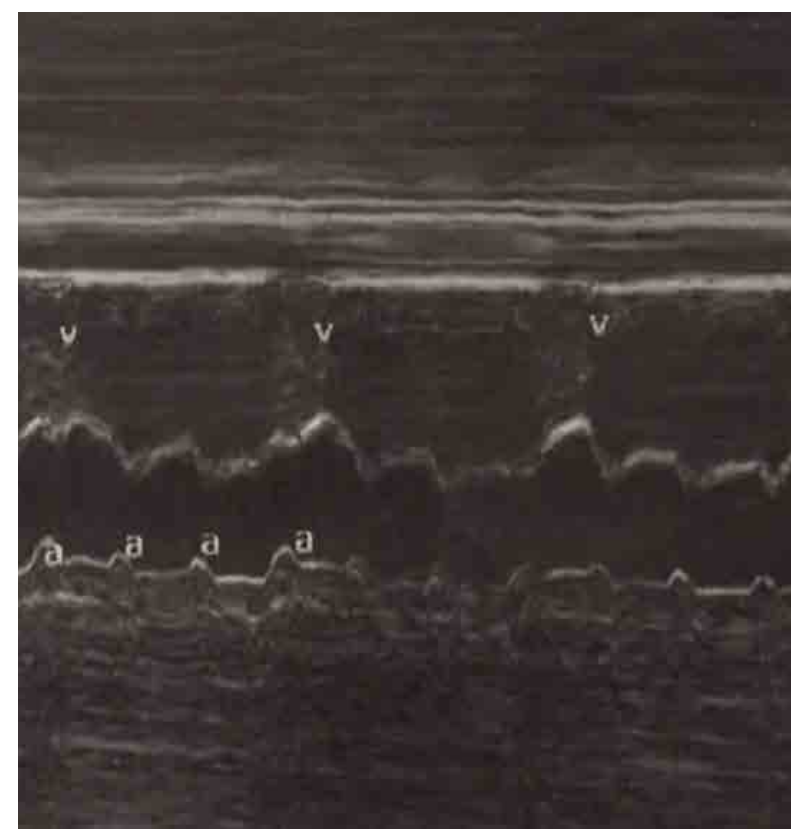

Figura 2. Fotografía tomada del ecocardiograma fetal realizado al ingreso a nuestra institución, con Modo M. Las letras "a" corresponden a la contracción auricular, las "v" a la contracción ventricular, con evidencia de disociación auriculoventricular, lo que corresponde a bloqueo auriculoventricular grado III o completo

logra frecuencia cardiaca fetal de $86 \mathrm{lpm}$, sin embargo, por la presencia de cefalea intensa, taquicardia y temblor en la madre es necesario disminuir la dosis a 2,5 mg vía oral cada 6 horas. Teniendo en cuenta la persistencia de frecuencia cardiaca fetal baja, la presencia de cardiopatía dilatada y el peso adecuado para la edad gestacional, a la semana 37 se decide finalizar la gestación mediante cesárea electiva.

En salas de cirugía se obtiene recién nacida vigorosa, con peso y talla adecuados para la edad gestacional; Ballard de 38 semanas, con adecuada adaptación neonatal a pesar de presentar frecuencia cardiaca de $50 \mathrm{lpm}$. Inicialmente sin signos de bajo gasto o de dificultad respiratoria. Aproximadamente a las 12 horas de vida la recién nacida presenta dificultad respiratoria, llenado capilar lento con tendencia a la hipotensión arterial, por lo cual se decide iniciar ventilación mecánica invasiva; se intenta mejorar la frecuencia cardiaca con medidas farmacológicas, sin respuesta de la paciente; se toma radiografía de tórax (figura 3) que muestra cardio- 


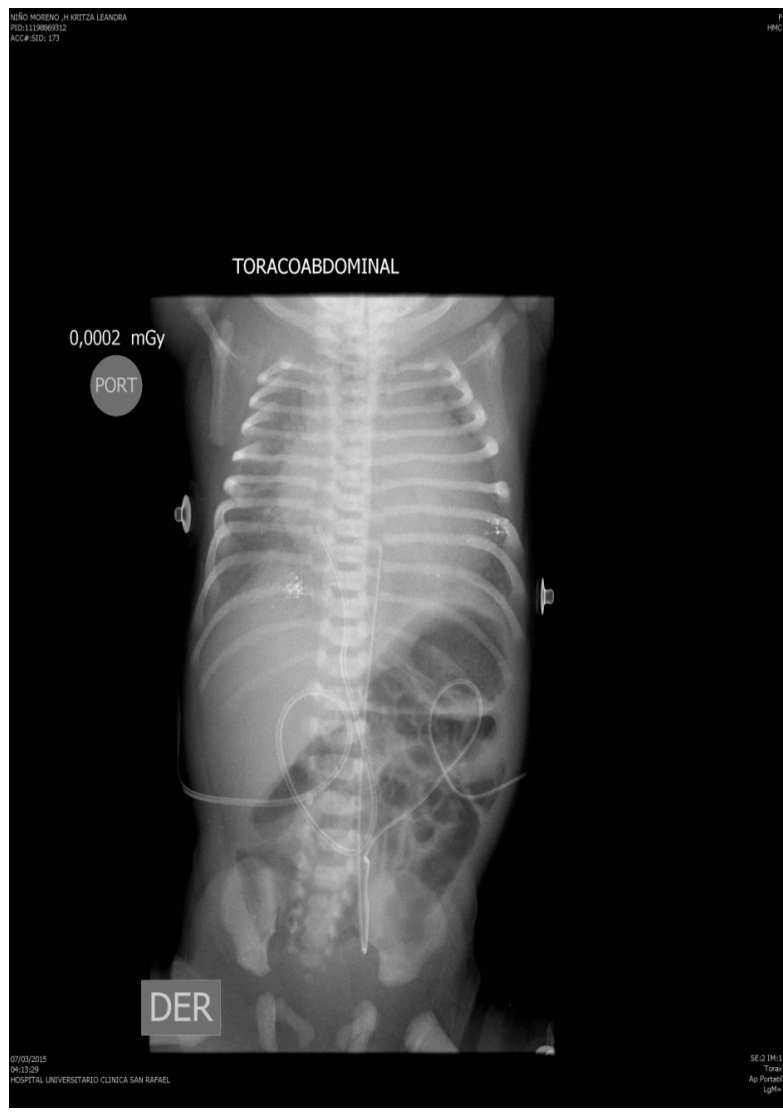

Figura 3. Radiografía de tórax de la recién nacida previo a la implantación del marcapasos. Se observa cardiomegalia

megalia. Se informa a cirugía cardiovascular quienes realizan implantación de marcapasos programado epicárdico unicameral ventricular, con frecuencia cardiaca de $120 \mathrm{lpm}$ y umbral de 0,9. Posterior a la cirugía la recién nacida es trasladada a la unidad de cuidados intensivos pediátricos para continuar manejo integral. En el ecocardiograma de control se evidencia marcapasos unicameral de demanda ventricular (VVI), con un corazón estructuralmente sano y adecuada función biventricular. La evolución de la recién nacida al año de vida ha sido satisfactoria, con adecuado desarrollo neurológico y ponderal.

\section{MATERIALES Y MÉTODOS}

Se realizó una revisión de la literatura publicada en las bases de datos: Medline vía PubMed, Lilacs y SciELO, utilizando los términos: "fetal complete atrioventricular block", "congenital complete heart block" y "congenital heart disease”. La búsqueda se limitó a trabajos publicados entre el año 2000 y enero de 2017, en inglés y español. Se incluyeron reportes, series de casos, cohortes y revisión de la literatura. Además, se realizó revisión con el término "Neonatal Lupus" en Up to Date.

Aspectos éticos. Se solicitó autorización verbal y escrita (mediante consentimiento informado) para la publicación del caso y la utilización del archivo fotográfico. Se garantizó la confidencialidad de la información y el anonimato de la paciente.

\section{RESULTADOS}

Se obtuvieron 34 publicaciones con los términos y límites descritos, de las cuales se seleccionaron 21. De estas, siete publicaciones corresponden a reportes de caso con revisión de la literatura realizadas en Estados Unidos (4, 5), Colombia (10), Alemania (14), Canadá y Estados Unidos (15), Australia (16) e Italia (17). Las otras 14 publicaciones seleccionadas corresponden a 9 revisiones de la literatura $(2,3,6$, $7,18-22)$ y a 5 estudios de cohorte: 3 retrospectivas $(8,11,13)$ y 2 prospectivas $(9,12)$. Sus enfoques fueron en pronóstico $(11,13)$, y diagnóstico y pronóstico $(8,9,12)$. Se realizaron en Estados Unidos (8), Italia (9), Finlandia (11), Canadá (12) y Estados Unidos (13).

Diagnóstico. El método más utilizado para el diagnóstico prenatal fue el ecocardiograma fetal doppler con el uso del modo My doppler pulsado, para determinar el intervalo PR, la relación auriculoventricular y la detección de anomalías intracardiacas (2-4). Dentro de los hallazgos ecocardiográficos fetales documentados Matta et al., en una revisión de la literatura, informan que la regurgitación valvular fue el hallazgo más frecuente $(63,2 \%)$, seguido por la hiperecogenicidad miocárdica/ valvular-fibroelastosis endocárdica en un 10,5\%, las contracciones atriales prematuras en un 7,9\% y, finalmente, efusión pericárdica en un 2,6\% (2). Estas alteraciones se desarrollan en el 1-2\% de los casos de bloqueo AV grado III, y producen una lesión informada como irreversible (8-10). Krishnan et al., en una revisión de la literatura, informan que 
tanto la regurgitación valvular como la fibroelastosis endocárdica preceden el bloqueo AV completo (8), llegando a alcanzar tasas de morbilidad del $67 \%$ y de mortalidad del $30 \%$ tanto en la vida fetal como en la posnatal $(9,18)$.

Una revisión narrativa (19) sugiere que una vez se ha documentado una bradiarritmia fetal es importante evaluar las características de la arritmia y determinar si existe compromiso hemodinámico. Además, propone realizar un ecocardiograma cada 8 días entre las semanas 16-28; en caso de ser normal se debe repetir desde la semana 28 cada 2-4 semanas.

Tratamiento. Los esteroides fluorados como la betametasona y dexametasona, al atravesar la barrera placentaria ingresan a la circulación fetal y mitigan la inflamación cardiaca provocada por los anticuerpos maternos mejorando la contractilidad cardiaca $(14,15,20,21)$, por lo que son los más utilizados ante bloqueos grados I y II según una revisión de la literatura (20). No obstante, esta misma revisión advierte que este grupo de medicamentos cada vez se utiliza menos debido a los reportes de efectos adversos fetales como insuficiencia adrenal, y neonatales como la alteración del crecimiento y del neurodesarrollo. Weber et al. recomiendan $8 \mathrm{mg} /$ día de dexametasona por dos semanas, posteriormente se disminuye la dosis a $4 \mathrm{mg}$ /día por dos semanas o se administran $2 \mathrm{mg} /$ día si el embarazo es mayor a 28 semanas (21). Se ha reportado mejoría en supervivencia por encima del $90 \%$ con el uso de beta agonistas (principalmente de terbutalina) en los casos de frecuencia cardiaca fetal menor a $55 \mathrm{lpm}$, ante la presencia de fibroelastosis, hidrops, o en casos en donde hay progresión del bloqueo $(14,20)$.

En 2011, Trucco et al. publicaron un reporte de 20 casos, en el cual administraron inmunoglobulina endovenosa (IGIV) asociada a corticoesteroides en gestantes cuyo feto cursaba con cardiomiopatía o fibroelastosis secundaria a bloqueo AV grado III, con buenos resultados y supervivencia mayor al $80 \%$ (15). La dosis recomendada de IGIV es de $400 \mathrm{mg} / \mathrm{kg} /$ día por 5 días, sin embargo, dadas las limitaciones de dicha publicación, se concluye que se requieren más estudios para determinar la dosis y duración adecuadas (15).

Sobre el manejo posnatal, Yang et al. reportan una serie de casos en el año 2012 en la cual analizaron una cohorte de 15 pacientes con bloqueo AV completo, y encontraron que el $87 \%$ de los recién nacidos requirió implantación de marcapasos como manejo definitivo (16). El momento de la implantación del marcapasos depende de si el paciente está sintomático, dado que la frecuencia ventricular de un bloqueo AV completo suele no ser suficiente para mantener el gasto cardiaco del neonato (22). Una frecuencia cardiaca menor a 55 lpm constituye una indicación absoluta para el uso de este tipo de aditamentos (22).

En los últimos años se han desarrollado marcapasos cada vez más pequeños y con baterías de mayor duración, lo cual prolonga el tiempo antes de una primera reintervención en este grupo de pacientes (16). En un reporte de casos con revisión de literatura se documenta que la tendencia es a implantar menos marcapasos unicamerales, prefiriendo los bicamerales (mayor dificultad técnica en el momento de la inserción), ya que ofrecen la ventaja de mayor respuesta sinusal y los beneficios hemodinámicos de la sincronía auriculoventricular (17).

En la vida posnatal diferentes estudios coinciden en que la mortalidad con un tratamiento temprano y adecuado oscila entre el 10-15\%, con una mortalidad del 5-8\% cuando se trata de bloqueo AV aislado y del 29-40\% cuando está asociado a enfermedad estructural $(16,17,22)$.

\section{CONCLUSIONES}

El bloqueo AV congénito de tercer grado es una entidad que, pese a ser poco frecuente, tiene un gran impacto y alto porcentaje de morbimortalidad perinatal. Al ser sus causas y diagnóstico fácilmente identificables, permite realizar un diagnóstico temprano y tratamiento oportuno, lo que constituye los pilares fundamentales en el abordaje de esta pato- 
logía. Aunque hay publicaciones con muy buenos resultados en cuanto al manejo fetal y neonatal, se requieren estudios con mayor calidad metodológica que aborden y permitan avalar otras opciones y esquemas terapéuticos prometedores.

\section{REFERENCIAS}

1. Gratacós Martínez G. Cardiología fetal. Madrid: Marbán; 2015.

2. Matta MJ, Cuneo BF. Doppler echocardiography for managing fetal cardiac arrhythmia. Clin Obstet Gynecol. 2010;53:899-914. https://doi.org/10.1097/ GRF.0b013e3181fbb747

3. Jaeggi ET, Friedberg M. Arrhythmias during pregnancy. Diagnosis and Management of Fetal Bradyarrhythmias. Pacing Clin Electrophysiol. 2008;31:S50-3. https:// doi.org/10.1111/j.1540-8159.2008.00957.x

4. DiMaio MA, Faix JD. Fetal atrioventricular heart block. Stanford University medical center. Clinical Chemistry. 2014;60:1153-7. https://doi.org/10.1373/ clinchem.2013.212035.

5. Lai J, Clark TJ, Tan JH, Delaney S, Jolley JA. Ultrasound findings in fetal congenital heart block associated with maternal anti-Ro/SSA and Anti-La/ SSB antibodies. Ultrasound Q.2015;31:34-6. https:// doi.org/10.1097/RUQ.0000000000000112.

6. Santos-Pardo I, Villuendas R, Salvador-Corres I, Martínez-Morillo M, Olivé A, Bayes-Genis A. Anti-Ro/SSA antibodies and cardiac rhythm disturbances: Present and future perspectives. Int J Cardiol. 2015;184:244-50. https://doi.org/10.1016/j. ijcard.2014.11.002

7. Buyon JP, Clancy RM. From antibody insult to fibrosis in neonatal lupus - The heart of the matter. 2003;5:266-70. https://doi.org/10.1186/ar763.

8. Krishnan A, Arya B, Moak JP, Donofrio MT. Outcomes of fetal echocardiographic surveillance in anti-SSA exposed fetuses at a large fetal cardiology center. Prenat Diagn. 2014;34:1207-12. https://doi. org/10.1002/pd.4454.

9. Brucato A, Frassi M, Franceschini F, Cimaz R, Faden D, Pisoni MP, et al. Risk of congenital complete heart block in newborns of mothers with anti-Ro/SSA antibodies detected by counterimmunoelectrophoresis: A prospective study of 100 women. Arthritis Rheum. 2001;44:1832-5. https://doi.org/ 10 .
1002/1529-0131(200108)44:8<1832::AID ART320>3.0.CO;2-C.

10. Díaz A, Serrano A, Guzmán M. Bloqueo aurículoventricular congénito completo. Reporte de un caso y revisión de la literatura. Rev Colomb Cardiol. 2008;15:35-42.

11. Eronen M, Sirèn MK, Ekblad H, Tikanoja T, Julkunen H, Paavilainen T. Short and Long-term outcome of children with congenital complete heart block diagnosed in utero or as a newborn. Pediatrics. 2000;106:86-91. https://doi.org/10.1542/ peds.106.1.86

12. Jaeggi E, Laskin C, Hamilton R, Kingdom J, Silverman E. The importance of the level of maternal antiRo/SSA antibodies as a prognostic marker of the development of cardiac neonatal lupus erythematosus. A prospective study of 186 antibody-exposed fetuses and infants. J Am Coll Cardiol. 2010;55:2778-84. https://doi.org/10.1016/j.jacc.2010.02.042.

13. Llanos C. Recurrence rates of cardiac manifestations associated with neonatal lupus and maternal/fetal risk factors, Manuscript A, Cytokines P, Transplantation P. Arthritis Rheum. 2010;9:1-14.

14. Hickstein H, Külz T, Claus R, Stange J, Schmidt R. Autoimmune-associated congenital heart block: Treatment of the mother with immunoadsorption. Ther Apher Dial. 2005;9:148-53. https://doi. org/10.1111/j.1774-9987.2005.00226.x.

15. Trucco SM, Jaeggi E, Cuneo B, Moon-Grady AJ, Silverman E, Silverman N, et al. Use of intravenous gamma globulin and corticosteroids in the treatment of maternal autoantibody-mediated cardiomyopathy. J Am Coll Cardiol. 2011;57:715-23. https://doi: 10.1016/j.jacc.2010.09.044.

16. Yan J, Varma SK, Malhotra A, Menahem S. Congenital complete heart block: Single tertiary center experience. Heart, Lung and Circulation. 2012;21:666-70. https://doi.org/10.1016/j.hlc.2012.05.784.

17. Ammiratti A, Silvetti MS, Di Carlo D, Saputo FA, Longoni A, Drago F. "De novo" biventricular pacing in two children with complete atrio-ventricular blockand severe ventricular dilatation: Early reverse remodeling. Int J Cardiol. 2012;160: e52-e53. https:// doi.org/10.1016/j.ijcard.2012.03.032.

18. Buyon JP, Clancy RM, Friedman DM. Autoimmune associated congenital heart block: Integration of clinical and research clues in the management 
of the maternal fetal dyad at risk. J Intern Med. 2009;265:653-62. https://doi.org/10.1111/j.13652796.2009.02100.x.

19. Buyon JP. Neonatal lupus: Epidemiology, pathogenesis, clinical manifestations, and diagnosis [visitado 2017 Nov 21]. Disponible en: https://www.uptodate.com/ contents/neonatal-lupus-epidemiology-pathogenesisclinical-manifestations-and-diagnosis.

20. Saxena A, Izmirly PM, Mendez B, Buyon JP, Friedman DM. Prevention and treatment in utero of autoimmune-associated congenital heart block.
Cardiol Rev. 2014;22:263-7. https://doi.org/10.1097/ CRD.0000000000000026

21. Weber R, Stambach D, Jaeggi E. Diagnosis and management of common fetal arrhythmias. J Saudi Heart Assoc.. 2011;23:61-6. https://doi. org/10.1016/j.jsha.2011.01.008

22. Baruteau A, Perry E, Sanatani S, Horie M, Dubin A. Evaluation and management of bradycardia in neonates and children. Eur J Pediatr. 2016; 175:15161. https://doi.org/10.1007/s00431-015-2689-z 